\title{
Effects of Cytochrome P450 Inhibition and Induction on the Phenotyping Metrics of the Basel Cocktail: A Randomized Crossover Study
}

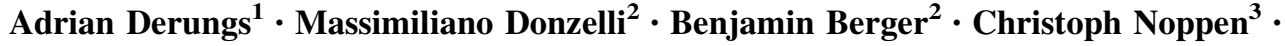 \\ Stephan Krähenbühl' ${ }^{2}$ Manuel Haschke ${ }^{2}$
}

Published online: 28 June 2015

(C) The Author(s) 2015. This article is published with open access at Springerlink.com

\begin{abstract}
Background and Objective Activity of human cytochrome P450 enzymes (CYPs) shows high inter-and intraindividual variability, which is determined by genetic and non-genetic factors. Using a combination of CYP-specific probe drugs, phenotyping cocktails allow simultaneous assessment of the activity of different CYP isoforms. The objective of this study was to characterize the phenotyping metrics of the Basel cocktail in healthy male subjects with induced and inhibited CYP activity.

Methods In a randomized crossover study, the probe drugs for simultaneous phenotyping of CYP1A2 (caffeine), CYP2B6 (efavirenz), CYP2C9 (losartan), 2C19 (omeprazole), CYP2D6 (metoprolol), and CYP3A4 (mi-
\end{abstract}

A. Derungs and M. Donzelli contributed equally to this article.

Electronic supplementary material The online version of this article (doi:10.1007/s40262-015-0294-y) contains supplementary material, which is available to authorized users.

Manuel Haschke

manuel.haschke@unibas.ch

1 Division of Clinical Pharmacology and Toxicology, Inselspital Bern, Bern, Switzerland

2 Division of Clinical Pharmacology and Toxicology, Departments of Biomedicine and Clinical Research, University Hospital Basel, Hebelstrasse 2, 4031 Basel, Switzerland

3 Viollier AG, Allschwil, Switzerland dazolam) were administered to 16 subjects without pretreatment (baseline), after pretreatment with a combination of CYP inhibitors (ciprofloxacin, ketoconazole, and paroxetine), and after CYP induction with rifampicin. All subjects were genotyped. Pharmacokinetic profiles of the probe drugs and their main metabolites and metabolic ratios $2,4,6$, and $8 \mathrm{~h}$ after probe drug application were determined in plasma and compared with the corresponding area under the plasma concentration-time curve (AUC) ratios.

Results The Basel phenotyping cocktail was well tolerated by all subjects independent of pretreatment. Good correlations of metabolic ratios with AUC ratios of the corresponding probe drugs and their metabolites for all three conditions (baseline, CYP inhibition, and CYP induction) were found at $2 \mathrm{~h}$ after probe drug administration for CYP3A4, at $4 \mathrm{~h}$ for CYP1A2 and CYP2C19, and at $6 \mathrm{~h}$ for CYP2B6 and CYP2D6. While CYP inhibition significantly changed AUC ratios and metabolic ratios at these time points for all six CYP isoforms, CYP induction did not significantly change AUC ratios for CYP2C9. For CYP3A4, total 1'-hydroxymidazolam concentrations after pretreatment of samples with $\beta$-glucuronidase were needed to obtain adequate reflection of CYP induction by the metabolic ratio.

Conclusions Inhibition of CYP activity can be detected with the Basel phenotyping cocktail for all six tested CYP isoforms at the proposed time points. The AUC ratio of losartan:losartan carboxylic acid in plasma does not seem suitable to detect induction of CYP2C9. The observed metabolic ratios for inhibited and induced CYP activity need to be confirmed for extensive metabolizers, and typical ratios for subjects with genetically altered CYP activity will need to be established in subsequent studies.

ClinicalTrials.gov-ID: NCT01386593. 


\section{Key Points}

Extensive validation data are required before new phenotyping cocktails can be introduced into clinical practice.

In this study, the phenotyping metrics of the recently introduced Basel phenotyping cocktail were characterized in healthy subjects with inhibited and induced cytochrome P450 enzyme (CYP) activity.

While CYP inhibition could be reliably detected for all tested isoforms, detection of CYP induction was more challenging, requiring e.g., the use of total $1^{\prime}$-hydroxymidazolam concentrations to obtain metabolic ratios that adequately reflect induced CYP3A4 activity.

\section{Introduction}

Human cytochrome P450 enzymes (CYPs) are responsible for oxidative metabolism of approximately $50 \%$ of commonly used drugs and many endogenous compounds. Interand intra-individual CYP activity is highly variable and determined by multiple factors, such as genetic variants, endogenous mediators, and environmental influences [1]. While genetic factors can be assessed by genotyping, the net effect of all influencing factors on CYP activity can only be assessed by phenotyping.

With the cocktail approach, the activity of several CYP isoforms can be simultaneously assessed by administration of a combination of CYP-specific probe drugs. Several phenotyping cocktails have been published to date. However, many have only been tested in subjects with normal CYP activity and data on the phenotyping metrics from systematic studies with inhibited and/or induced CYP function are only available for some of these cocktails [26].

Studying a phenotyping cocktail in subjects with altered CYP activity is an essential part of the validation process. CYP induction or inhibition will lead to very low concentrations of the probe drug or its metabolite, and highly sensitive bioanalytical methods are required to obtain a reliable phenotyping metric. Such studies will also provide a typical range of values for low or high CYP activity for each phenotyping metric, which can serve as a reference for future applications of the cocktail.

We recently have developed a new phenotyping cocktail for six major CYP isoforms [7]. Our cocktail is based on low-dose probe drugs that are widely used in clinical practice and easily available on the market. We showed that the new combination of probe drugs can be used without mutual interactions and without causing relevant adverse effects. In subjects without inhibited or induced CYP activity, metabolic ratios (i.e., concentration ratio of probe drug and CYP-specific metabolite) determined in a single blood sample correlated well with area under the plasma concentration-time curve (AUC) ratios of the probe drugs and their metabolites. The goal of the current study was to characterize the phenotyping metrics of our cocktail in healthy male subjects after pretreatment with a combination of CYP inhibitors and a CYP inducer. This is an important step in the ongoing validation process of this phenotyping cocktail.

\section{Methods}

\subsection{Clinical Study}

A single-center, randomized, two-way crossover study (ClinicalTrials.gov ID: NCT01386593) was performed at the Phase I Research Unit, University Hospital Basel, Switzerland. Healthy, male non-smoking Caucasian subjects without clinically significant findings on physical examination, routine laboratory, and electrocardiogram (ECG) evaluations were included to limit confounding influences of ethnicity and sex on drug metabolism. Use of herbal medicines, over-the-counter medications, or food products known to be inducers or inhibitors of CYP450 (e.g., grapefruit juice) was forbidden during the entire study. Consumption of alcoholic- and caffeine-containing beverages was not allowed for $72 \mathrm{~h}$ prior to dosing until after the last sampling time point of the study period. Probe drugs of the Basel cocktail [7] (100 mg caffeine, $50 \mathrm{mg}$ efavirenz, $12.5 \mathrm{mg}$ losartan, $10 \mathrm{mg}$ omeprazole, $12.5 \mathrm{mg}$ metoprolol, and $2 \mathrm{mg}$ midazolam) were administered alone (baseline), after pretreatment with a combination of three CYP inhibitors at clinically recommended doses (fluconazole 400-mg loading dose, followed by $200 \mathrm{mg}$ per day, ciprofloxacin $750 \mathrm{mg}$ every $12 \mathrm{~h}$, and paroxetine $20 \mathrm{mg}$ per day), and after CYP induction with rifampicin (600 mg once daily for 7 days). All subjects first received the baseline assessment (study period 1), followed by the two assessments after CYP inhibition or CYP induction (study periods 2 and 3 ) in a randomized order. After the baseline assessment, wash-out time was 14 days, and between the second and third study period, 21 days allowed sufficient time for elimination of all probe drugs after CYP inhibition.

CYP inhibitors were selected from a list of recommended in vivo CYP inhibitors based on their inhibitory potency [8]. Ciprofloxacin is a strong inhibitor ( $\geq 5$-fold 
increase in AUC or $>80 \%$ decrease in clearance) of CYP1A2, fluconazole is a strong inhibitor of CYP2C19 and a moderate inhibitor ( $\geq 2$ but $<5$-fold increase in AUC or 50-80 \% decrease in clearance) of CYP2C9 and CYP3A4. Paroxetine is a strong inhibitor of CYP2D6. For CYP2B6, no strong inhibitors approved for human use are available and paroxetine, which has been shown to be a CYP2B6 inhibitor in vitro [9], was used. Paroxetine is mainly metabolized by CYP2D6 with minor metabolism by CYP3A4 and CY1A2. As only minor pathways are inhibited by fluconazole and ciprofloxacin, no relevant effects on paroxetine exposure are expected. As all three CYP inhibitors have the potential to increase the corrected QT interval, 12-lead ECGs were recorded $1 \mathrm{~h}$ before and 2 and $12 \mathrm{~h}$ after probe drug intake.

Rifampicin is a strong inducer $(\geq 80 \%$ decrease in AUC) of CYP3A, a moderate inducer (50-80\% decrease in AUC) of CYP2B6, 2C9, and 2C19, and a weak inducer of CYP1A2 [3]. For CYP2D6, no inducer has been described to date. Duration of pretreatment with CYP inhibitors was selected to achieve more than $90 \%$ of steady-state concentrations before administration of the cocktail probe drugs. Duration of pretreatment with rifampicin was selected based on clinical data, indicating that a significant inducing effect is reached after 7 days [10].

Fluconazole and paroxetine were given in the morning on days $-3,-2$, and -1 , ciprofloxacin in the morning and in the evening of day -1 , and a last dose of each inhibitor was given in the morning of the study day, $1 \mathrm{~h}$ before administration of the phenotyping cocktail probe drugs. Each treatment was investigated in the same group of 16 healthy male subjects.

Blood samples for pharmacokinetic analysis were collected before dosing and $0.25,0.50,0.75,1,2,3,4,6,8$, $12,24,48$, and $72 \mathrm{~h}$ after drug administration from an antecubital vein into ethylenediaminetetraacetic acid tubes. The tubes were centrifuged $\left(1500 \mathrm{~g}\right.$ for $10 \mathrm{~min}$ at $\left.4{ }^{\circ} \mathrm{C}\right)$ and plasma was stored at $-80^{\circ} \mathrm{C}$ until analysis. To assess compliance of the study subjects with the CYP inhibitor and inducer pretreatments, concentrations of ciprofloxacin, paroxetine, fluconazole, and rifampicin were measured in aliquots of the plasma samples collected for pharmacokinetic analysis. Oral fluid and dried blood spot samples were collected at the same time points as the blood samples. Results of these analyses will be presented elsewhere. Pharmacodynamic assessments (heart rate, blood pressure, sedation score) were performed at baseline and 1, 2, 4, 6, and $12 \mathrm{~h}$ after drug administration. Subjects rated their level of sedation on a visual analog scale with a range from 0 , "not tired" to 10, "very tired". All adverse events regardless of severity or relationship to the study drug were documented throughout the study.

\subsection{Study Drugs}

Caffeine (Coffeinum ${ }^{\circledR}$ N 0.2 g, Mylan Dura $\mathrm{GmbH}$, Darmstadt, Germany), efavirenz (Stocrin ${ }^{\circledR}$, Merck Sharp \& Dohme-Chibret AG, Opfikon, Switzerland), losartan $\left(\right.$ Cosaar $^{\circledR}$, Merck Sharp \& Dohme-Chibret AG, Opfikon, Switzerland), metoprolol (Belok ZOK ${ }^{\circledR}$, AstraZeneca AG, Zug, Switzerland), omeprazole (Antramups ${ }^{\circledR}$, AstraZeneca AG, Zug, Switzerland), midazolam (midazolam oral solution, $2 \mathrm{mg} / \mathrm{mL}$, University Hospital Pharmacy, Basel, Switzerland), ciprofloxacin (Ciproxin ${ }^{\circledR}, 750 \mathrm{mg}$, Bayer AG, Zurich, Switzerland), fluconazole (Diflucan ${ }^{\circledR}, 200 \mathrm{mg}$, Pfizer AG, Zurich, Switzerland), paroxetine (Deroxat ${ }^{\circledR}$, 20 mg, GlaxoSmithKline, Münchenbuchsee, Switzerland), and rifampicin (Rimactan ${ }^{\circledR}, 600 \mathrm{mg}$, Sandoz Pharmaceuticals, Rotkreuz, Switzerland) were purchased through the University Hospital Pharmacy, Basel, Switzerland.

\subsection{Materials and Reagents}

Caffeine-d9, ciprofloxacin, efavirenz-d4, 8-hydroxyefavirenz, 8,14-dihydroxyefavirenz, fluconazole, losartan, losartan carboxylic acid (E3174), losartan-d4, $\alpha$-hydroxymetoprolol, metoprolol-d7, omeprazole, 5-hydroxyomeprazole, omeprazole-d3, paroxetine, and rifampicin were purchased from Toronto Research Chemicals (Toronto, Canada). 1'-Hydroxymidazolam and midazolam-d6 were purchased from Lipomed (Lipomed AG, Arlesheim, Switzerland). Metoprolol, paraxanthine, and $\beta$-glucuronidase were obtained from Sigma-Aldrich (SigmaAldrich Chemie GmbH, Buchs, Switzerland). Midazolam (Hoffmann-La Roche, Basel, Switzerland) and efavirenz (Merck, NJ, USA) were kindly provided by the producers.

Formic acid, high-performance liquid chromatography (HPLC)-grade methanol, and water were purchased from Merck (Merck, Darmstadt, Germany). Stock solutions, calibration spiking solutions, and quality controls were prepared in dimethyl sulfoxide. Calibration standards were prepared by enriching caffeine-free blank human serum using the corresponding spiking solutions. Internal standard solutions containing the deuterated compounds were prepared in methanol.

\subsection{Bioanalysis}

Concentrations of cocktail probe drugs and their main metabolites as well as concentrations of the CYP inhibitors ciprofloxacin, ketoconazole, paroxetine, and the CYP inducer rifampicin in plasma samples were determined by reversed phase HPLC tandem mass spectrometry as described previously [7] with some modifications. Fifty microliter aliquots of plasma were mixed with $150 \mu \mathrm{L}$ internal standard solution containing deuterated analogs of 
each of the six cocktail probe drugs at a concentration of $100 \mathrm{ng} / \mathrm{mL}$, vortex mixed for $30 \mathrm{~s}$, and centrifuged $(3220 \mathrm{~g}$, $\left.30 \mathrm{~min}, 10^{\circ} \mathrm{C}\right)$. Chromatographic separation was performed on a Shimadzu HPLC system (Shimadzu AG, Reinach, Switzerland) coupled to a triple quadrupole tandem mass spectrometer (API4000, AB/MDS Sciex, Concord, Canada) operating in positive electrospray ionization mode, except for efavirenz and its metabolite, which were detected in negative mode. Samples were eluted on an Atlantis T3 column $(2.1 \times 50 \mathrm{~mm}, 3 \mu \mathrm{m}$, Waters, BadenDättwil, Switzerland). The injection volume was $10 \mu \mathrm{L}$ and total run time was $2.5 \mathrm{~min}$. The following gradient was applied: from 0 to $0.5 \mathrm{~min} 100 \%$ solvent $\mathrm{A}(0.1 \%$ formic acid in water), from 0.5 to $2 \mathrm{~min}$ the proportion of solvent B $(0.1 \%$ formic acid in methanol) linearly increased from 50 to $70 \%$, at $2.01 \mathrm{~min}$, the percentage of B was increased to $95 \%$ and maintained for $0.5 \mathrm{~min}$. At $2.5 \mathrm{~min}$, the initial conditions were re-established. The flow rate was $0.8 \mathrm{~mL} /$ min, the column was placed in a column oven at $60{ }^{\circ} \mathrm{C}$, and the samples were stored in the autosampler at $10^{\circ} \mathrm{C}$. A calibration curve was constructed, plotting analyte concentration vs. peak area ratios of the analyte/deuterated internal standard measured in plasma standard samples. Deuterated analogs of each cocktail probe drug were used for the respective parent drug and metabolite. For fluconazole, paroxetine, ciprofloxacin, and rifampicin, midazolam-d6 was used as the internal standard. Inter-assay accuracy (determined as the percent bias) for plasma quality control samples ranged from -11.6 to $10.2 \%$ and inter-assay precision (determined as the relative standard deviation) was lower than $10.4 \%$ for all analytes (Supplementary Table 1). The lower limit of quantification was $0.5 \mathrm{ng} / \mathrm{mL}$ for $\alpha$-hydroxymetoprolol, omeprazole, 5-hydroxyomeprazole, midazolam, 1'-hydroxymidazolam, and 8,14-dihydroxyefavirenz, $1 \mathrm{ng} / \mathrm{mL}$ for metoprolol, losartan, losartan carboxylic acid, efavirenz, 8-hydroxyefavirenz, paroxetine, and rifampicin, $10 \mathrm{ng} / \mathrm{mL}$ for paraxanthine and caffeine, $25 \mathrm{ng} / \mathrm{mL}$ for fluconazole, and $50 \mathrm{ng} / \mathrm{mL}$ for ciprofloxacin. Calibration ranges and autosampler stability data are given in Supplementary Tables 2 and 3. For all 1'-hydroxymidazolam measurements, $50-\mu \mathrm{L}$ aliquots of plasma were incubated with 500 units of $\beta$-glucuronidase Type HP 2 (Sigma G7017, SigmaAldrich, Buchs, Switzerland) at $37^{\circ} \mathrm{C}$ for $16 \mathrm{~h}$. Further sample processing was the same as described for not preincubated plasma samples.

\subsection{Data Analysis}

Areas under the plasma concentration time curve (AUC) from time 0 to $24 \mathrm{~h}$ after dosing $\left(\mathrm{AUC}_{24}\right)$ or from time 0 to the last quantifiable concentration if the last quantifiable concentration was observed before $24 \mathrm{~h}$ after dosing were estimated with non-compartmental methods using Phoenix WinNonlin software (Certara, Princeton, NJ, USA). AUCs after inhibition or induction were compared with baseline AUCs for all parent drugs and their metabolites using a WinNonlin average bioequivalence model. Correlations between single time point metabolic ratios (i.e., parent drug concentration over metabolite concentration, except for CYP1A2 where the inverse ratio was used) and corresponding $\mathrm{AUC}_{\text {parent }} / \mathrm{AUC}_{\text {metabolite }}$ ratios were analyzed using Spearman's rank correlations. $\mathrm{AUC}_{\text {parent }} / \mathrm{AUC}_{\text {metabo- }}$ lite ratios and metabolic ratios after CYP inhibition and CYP induction were compared with baseline ratios using Wilcoxon signed rank tests with a corrected $p<0.025$ for the two comparisons. Concentration ratios of extensive, intermediate, and poor metabolizers were compared using nonparametric Kruskal-Wallis tests. For CYP1A2, 2B6, 2C9, and 2C19 subjects with two wild-type alleles (*1A or *1) and for CYP2D6 with at least one fully functional allele $(* 1$ or $* 2$ ) were considered as extensive metabolizers [11-15]. Pharmacodynamic parameters (heart rate, blood pressure, sedation scores) were parametrized by calculating area under the effect curves from time 0 to $12 \mathrm{~h}$ and analyzed using a one-way, repeated-measures analysis of variance after testing for normal distribution. IBM SPSS Statistics, version 21 (IBM Corporation, Armonk, NY, USA) was used for statistical analyses.

\subsection{Genotype Analysis}

DNA was isolated from ethylenediaminetetraacetic acid blood on an m2000sp instrument (Abbott Molecular, De Plaines, IL, USA). Specific single nucleotide polymorphisms in the genes encoding CYP1A2 (alleles *1A, *1F), CYP2B6 (allele*6), CYP2C9 (alleles *2, *3), CYP2C19 (alleles $* 2, * 3$ ), and CYP2D6 (alleles *3, *4, *6) were detected using primer/probe real-time polymerase chain reaction (PCR) reagents (Lightmix, TIB MOLBIOL, Berlin, Germany) on a Lightcycler 1.5 instrument (Roche Diagnostics, Rotkreuz, Switzerland) [16, 17]. For detection of CYP2D6 allele *5 (gene deletion) and *XN (gene duplication), a long-range PCR using the Expand Long Template PCR system kit (Roche Diagnostics) was used. For detection and confirmation of CYP2D6 rare alleles $(* 41, * X N)$, hybridization of amplified products was performed using the DNA chip technology (PharmaChip, Progenika, Derio, Spain).

\section{Results}

Sixteen healthy male volunteers (mean age 23.8 years, range 20-35 years, mean body mass index $23.5 \mathrm{~kg} / \mathrm{m}^{2}$, range $20.6-27.5 \mathrm{~kg} / \mathrm{m}^{2}$ ) completed the study according to 
the protocol. All subjects had no history of relevant disease or drug abuse. All study subjects were of Caucasian ethnicity, with normal findings on physical examination, normal screening laboratory tests (including screening for drugs of abuse), and normal electrocardiograms. The phenotyping probe drugs of the Basel cocktail, as well as the drugs used for CYP inhibition and induction were tolerated without serious adverse events. No clinically or statistically significant changes in heart rate, blood pressure, or sedation scores compared with baseline were observed (Supplementary Fig. 1). No clinically relevant changes of electrocardiogram parameters were observed. After administration of the CYP inhibitor cocktail, all corrected QT intervals remained below $450 \mathrm{~ms}$.

Compliance with the inhibitor and inducer pretreatment was assessed by determination of the AUCs of ciprofloxacin, paroxetine, fluconazole, and rifampicin (Supplementary Fig. 2). All 16 study participants showed adequate exposure to the three CYP inhibitors. However, data from one study participant had to be excluded from analysis of the induction data. This subject had rifampicin concentrations approximately 100-fold higher compared with the other subjects, either owing to an erroneous intake of rifampicin shortly before application of the cocktail probe drugs or non-compliance with the pretreatment regimen and insufficient auto-induction of rifampicin metabolism.

The plasma concentration-time profiles for the six probe drugs of the Basel cocktail and their metabolites are shown in Fig. 1. Compared with baseline conditions, pretreatment with the three CYP inhibitors increased the concentrations of the parent probe drugs (except for CYP2B6) and decreased the concentrations of their respective metabolites (except for CYP3A4). Pretreatment with the CYP inducer rifampicin had the opposite effect on the parent probe drug concentrations (except for CYP2D6), while the concentrations of the metabolites only increased for CYP2B6. For the other CYP isoforms, the metabolite concentrations after induction were either unchanged or lower compared with baseline.

Geometric mean AUC ratios and metabolic ratios for 2, 4,6 , and $8 \mathrm{~h}$ after probe drug administration for each CYP isoform are shown in Table 1. AUC ratios after pretreatment with CYP inhibitors were significantly different from baseline AUC ratios for all CYP isoforms. After CYP induction with rifampicin, a significant change of AUC ratios was observed for all $\mathrm{CYP}$ isoforms except for CYP2C9, where the losartan:E3174 AUC ratio did not adequately reflect induced CYP activity.

The metabolic ratios in plasma were highly correlated with the corresponding AUC ratios for most of the tested time points (Table 2). The highest overall correlation between metabolic ratios and AUC ratios for all three conditions combined was found at $2 \mathrm{~h}$ after probe drug administration for CYP3A4, at $4 \mathrm{~h}$ for $\mathrm{CYP} 1 \mathrm{~A} 2$ and CYP2C19, and at $6 \mathrm{~h}$ for CYP2B6, CYP2C9, and CYP2D6.

Inhibition increased metabolic ratios (higher parent and lower metabolite concentration) for all CYP isoforms except for CYP1A2 (Fig. 2). For this isoform, the inverse ratio (i.e., metabolite over parent concentration) was used for better comparability with published data; and this inverse ratio decreased compared with baseline. The effect of CYP inhibition could be detected in almost every study subject for all tested CYP isoforms. After CYP inhibition, metabolic ratios were significantly different from baseline for all tested time points (i.e., with data available for inhibition and induction) for CYP1A2, 2B6, 2C9 (except for the 2-h ratio), 2C19, and 2D6.

After CYP induction, metabolic ratios were significantly different from baseline for all tested time points for CYP1A2, 2B6, and 2C19 (Table 1; Fig. 3). For CYP2D6, no consistent difference of metabolic ratios compared with baseline was observed. Because of induced glucuronidation of $1^{\prime}$-hydroxymidazolam, which quantitatively outweighed the induced formation of $1^{\prime}$-hydroxymidazolam [18], the metabolic ratio for CYP3A only was significantly different from baseline when total 1'-hydroxymidazolam concentrations after pretreatment of samples with $\beta$-glucuronidase were used. After deglucuronidation, total 1'-hydroxymidazolam concentrations were approximately 8 - to 10 -fold higher compared with $1^{\prime}$-hydroxymidazolam concentrations before deglucuronidation (Supplementary Table 4). In contrast to CYP3A4, deglucuronidation did not improve metabolic ratios for induction of CYP2B6 (data not shown). The concentrations of 8,14-dihydroxy-efavirenz, a secondary CYP2B6-mediated metabolite of 8-hydroxyefavirenz, were below the limit of quantification and lower than $6 \mathrm{ng} / \mathrm{mL}$ after deglucuronidation. Therefore, this dihydroxymetabolite was not considered for further analyses.

One subject did not consent to genotype testing; all other study participants were genotyped for the most important altered-function alleles (Supplementary Table 5). CYP2D6 genotyping identified one intermediate metabolizer (IM) with a combination of a loss-of-function and a diminished-function allele (CYP2D6*4/41) and 14 extensive metabolizers (EM) with at least one fully functional allele $(* 1$ or $* 2)$ [15]. Without pre-treatment, the CYP2D6 IM had a three-fold higher metabolic ratio at $6 \mathrm{~h}$ compared with EMs (8.5 vs. median value of EMs 2.3). Pretreatment with paroxetine increased the metabolic ratios of all subjects regardless of genotype and increased metabolic ratios of EM subjects to the range observed for the non-inhibited IM (Fig. 2e).

For CYP2B6, no subject was homozygous and nine subjects were heterozygous for a diminished-function allele of CYP2B6 (CYP2B6*6). Although the subject with 

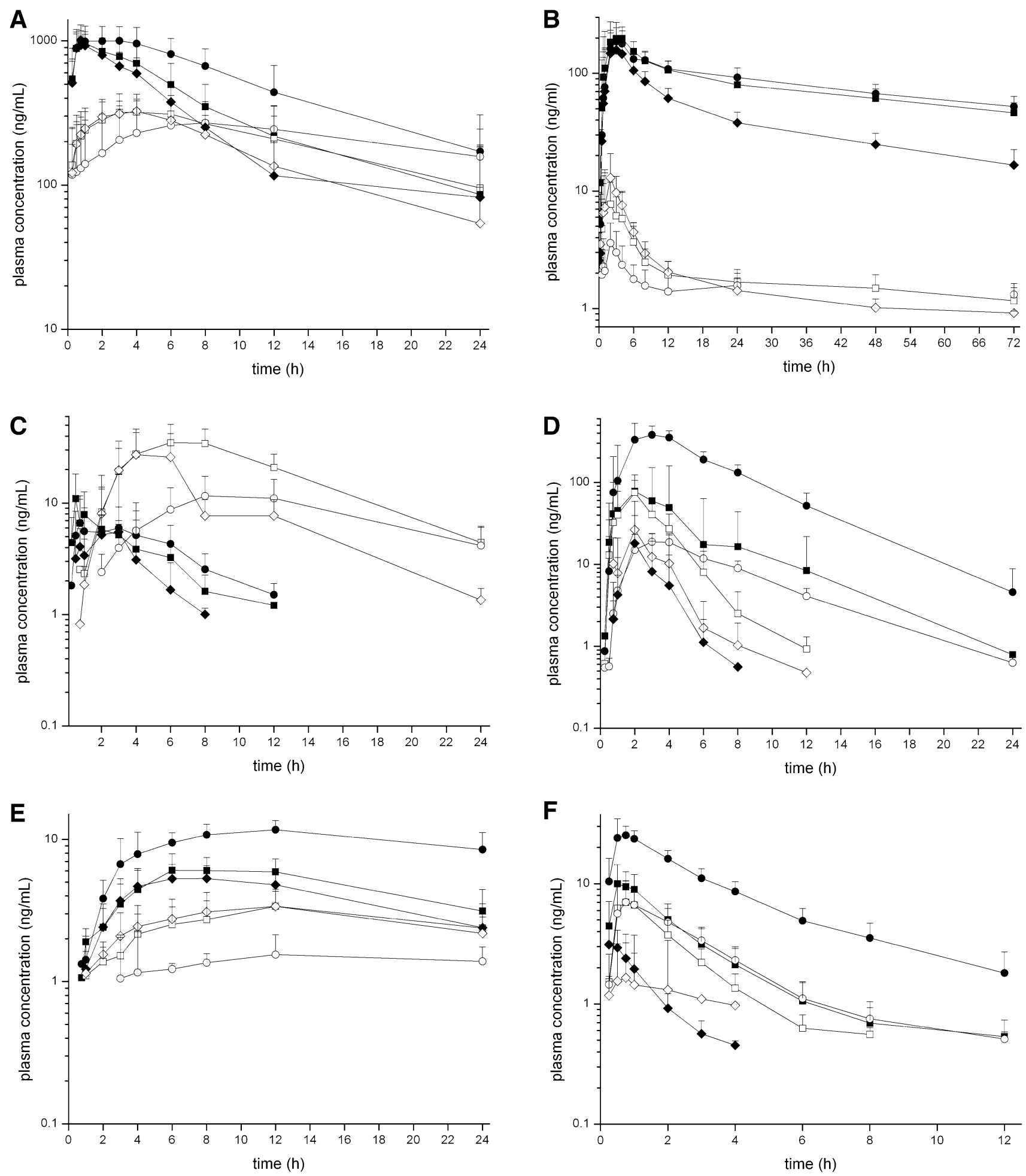

Fig. 1 Plasma concentration-time profiles of the Basel cocktail probe drugs under baseline conditions (squares), after pretreatment with a combination of CYP inhibitors (circles) and after pretreatment with the CYP inducer rifampicin (diamonds). Concentration-time profiles are shown for the probe drugs (closed symbols, solid lines) and their respective metabolites (open symbols, dashed lines). a Caffeine and paraxanthine (CYP1A2), b efavirenz and 8-hydroxyefavirenz (CYP2B6), c losartan and losartan carboxylic acid (CYP2C9),

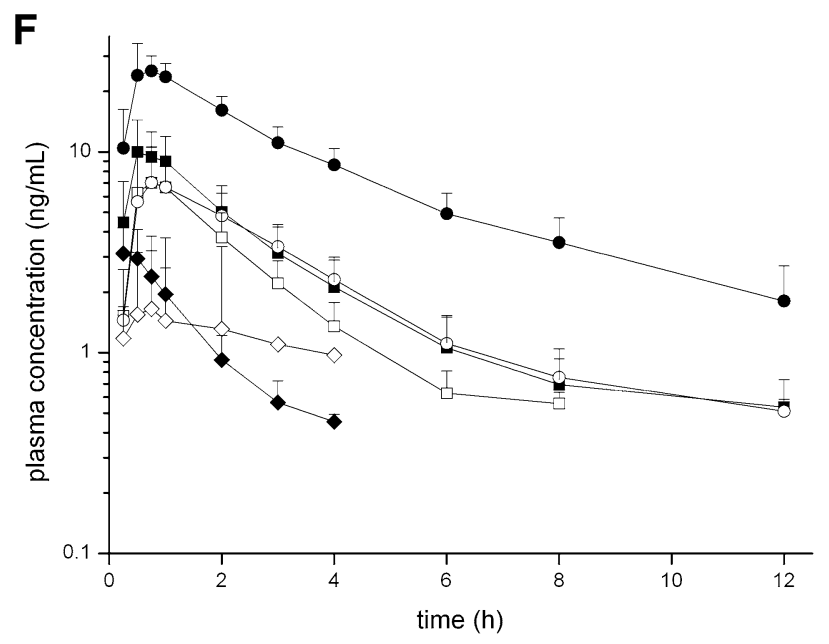

d omeprazole and 5-hydroxyomeprazole (CYP2C19), e metoprolol and $\alpha$-hydroxymetoprolol (CYP2D6), $\mathbf{f}$ midazolam and $1^{\prime}$-hydroxymidazolam (CYP3A4). Pretreatment with a combination of CYP inhibitors significantly changed the pharmacokinetic profiles of all probe drugs and their metabolites while the CYP inducer rifampicin predominantly affected the probe drugs for CYP2B6, 2C19, and 3A4. CYP cytochrome P450 enzyme 
Table 1 AUC ratios and metabolic ratios after administration of cocktail probe drugs with and without inhibitors and inducer

\begin{tabular}{|c|c|c|c|c|c|}
\hline CYP & Metric & Time (h) & Cocktail alone & Cocktail + inhibitors & Cocktail + inducer \\
\hline \multirow[t]{5}{*}{$1 \mathrm{~A} 2$} & $\mathrm{AUC}_{24} \mathrm{par} / \mathrm{AUC}_{24} \mathrm{caf}$ & & $0.63(0.58-0.71)$ & $0.38(0.35-0.45)^{* * *}$ & $0.64(0.59-0.70)^{* * * a}$ \\
\hline & {$[\mathrm{par}] /[\mathrm{caf}]$} & 2 & $0.32(0.29-0.38)$ & $0.13(0.11-0.20)^{* * * b}$ & $0.38(0.35-0.44)^{* * * a}$ \\
\hline & {$[\mathrm{par}] /[\mathrm{caf}]$} & 4 & $0.46(0.41-0.55)$ & $0.21(0.18-0.27)^{* * *}$ & $0.57(0.52-0.64)^{* * * a}$ \\
\hline & {$[\mathrm{par}] /[\mathrm{caf}]$} & 6 & $0.64(0.58-0.79)$ & $0.30(0.26-0.38)^{* * * a \mathrm{a}}$ & $0.78(0.72-0.89)^{* * * a}$ \\
\hline & {$[\mathrm{par}] /[\mathrm{caf}]$} & 8 & $0.80(0.71-0.97)$ & $0.39(0.35-0.49)^{* * *}$ & $0.96(0.87-1.13) * * *^{\mathrm{a}}$ \\
\hline \multirow[t]{5}{*}{ 2B6 } & $\mathrm{AUC}_{24} \mathrm{efa} / \mathrm{AUC}_{24} \mathrm{OH}-\mathrm{efa}$ & & $41.7(37.9-48.2)$ & $73.9(62.2-99.1)^{* * *}$ & $21.1(19.7-23.2)^{* * * a}$ \\
\hline & {$[\mathrm{efa}] /[\mathrm{OH}-\mathrm{efa}]$} & 2 & $23.7(21.1-29.0)$ & $51.2(43.3-70.0)^{* * *} \mathrm{~b}$ & $12.8(10.7-15.0)^{* * * a \mathrm{a}}$ \\
\hline & {$[\mathrm{efa}] /[\mathrm{OH}-\mathrm{efa}]$} & 4 & $38.7(34.8-48.5)$ & $84.1(71.9-115.0)^{* * *}$ & $19.3(17.9-21.3)^{* * *^{\mathrm{a}}}$ \\
\hline & {$[\mathrm{efa}] /[\mathrm{OH}-\mathrm{efa}]$} & 6 & $43.7(39.5-51.3)$ & $77.6(69.3-94.2)^{* * * *^{\mathrm{a}}}$ & $23.1(21.3-25.7)^{* * *^{\mathrm{a}}}$ \\
\hline & {$[\mathrm{efa}] /[\mathrm{OH}-\mathrm{efa}]$} & 8 & $53.2(47.8-62.6)$ & $88.7(79.7-108.0)^{* * *}$ & $27.7(26.0-30.8)^{* * * a \mathrm{a}}$ \\
\hline \multirow[t]{5}{*}{$2 \mathrm{C} 9$} & AUClos/AUCE3174 & & $0.09(0.06-0.15)$ & $0.24(0.19-0.41)^{* * *}$ & $0.07(0.06-0.11) \mathrm{ns}^{\mathrm{a}}$ \\
\hline & {$[\operatorname{los}] /[\mathrm{E} 3174]$} & 2 & $1.18(1.17-2.99)$ & $3.39(2.66-4.62) \mathrm{ns}^{\mathrm{f}}$ & $0.44(0.37-0.96)^{* * \mathrm{~b}}$ \\
\hline & {$[\operatorname{los}] /[\mathrm{E} 3174]$} & 4 & $0.17(0.14-0.37)$ & $1.17(1.03-2.09)^{* * *}$ & $0.08(0.06-0.16)^{* * a}$ \\
\hline & {$[\operatorname{los}] /[\mathrm{E} 3174]$} & 6 & $0.10(0.08-0.19)$ & $0.55(0.47-1.01)^{* * * *^{\mathrm{a}}}$ & $0.06(0.04-0.12) * * * \mathrm{c}$ \\
\hline & {$[\operatorname{los}] /[\mathrm{E} 3174]$} & 8 & $0.05(0.04-0.08)$ & $0.24(0.20-0.42)^{* * *}$ & $0.05(0.04-0.07) \mathrm{ns}^{\mathrm{b}}$ \\
\hline \multirow[t]{5}{*}{$2 \mathrm{C} 19$} & AUCome/AUCOH-ome & & $0.89(0.76-1.17)$ & $17.9(16.0-21.3)^{* * *}$ & $0.22(0.19-0.30)^{* * * a}$ \\
\hline & [ome]/[OH-ome] & 2 & $1.08(0.90-1.46)$ & $25.8(22.7-33.2) * * *$ & $0.25(0.22-0.35)^{* * a}$ \\
\hline & [ome]/[OH-ome] & 4 & $0.49(0.38-0.86)$ & $18.1(16.0-22.5)^{* * *}$ & $0.18(0.15-0.24)^{* * * a}$ \\
\hline & [ome]/[OH-ome] & 6 & $0.28(0.18-0.55)^{\mathrm{b}}$ & $14.6(12.5-18.8) \mathrm{nt}^{\mathrm{a}}$ & na \\
\hline & [ome]/[OH-ome] & 8 & na & na & na \\
\hline \multirow[t]{5}{*}{ 2D6 } & $\mathrm{AUC}_{24} \mathrm{met} / \mathrm{AUC}_{24} \mathrm{OH}-\mathrm{met}$ & & $1.69(1.54-1.93)$ & $7.57(5.48-10.89)^{* \mathrm{~g}}$ & $1.45(1.34-1.62)^{* * * \mathrm{~b}}$ \\
\hline & {$[\mathrm{met}] /[\mathrm{OH}-\mathrm{met}]$} & 2 & $1.93(1.56-2.47)^{\mathrm{e}}$ & na & $1.83(1.60-2.18) \mathrm{nt}^{\mathrm{d}}$ \\
\hline & {$[\mathrm{met}] /[\mathrm{OH}$-met $]$} & 4 & $2.20(2.00-2.54)$ & $6.60(5.90-7.85)^{* * *}$ & $2.02(1.84-2.31) \mathrm{ns}^{\mathrm{a}}$ \\
\hline & {$[\mathrm{met}] /[\mathrm{OH}-\mathrm{met}]$} & 6 & $2.36(2.16-2.69)^{\mathrm{a}}$ & $8.99(8.33-10.01)^{* * *}$ & $1.88(1.69-2.20)^{*^{\mathrm{a}}}$ \\
\hline & {$[\mathrm{met}] /[\mathrm{OH}$-met $]$} & 8 & $2.11(1.91-2.44)$ & $9.90(9.06-11.39)^{* * *}$ & $1.67(1.51-1.93) \mathrm{ns}^{\mathrm{b}}$ \\
\hline \multirow[t]{5}{*}{$3 \mathrm{~A} 4$} & AUCmid/AUC1-OH-mid & & $1.55(1.36-2.08)$ & $4.44(3.92-5.67)^{* * *}$ & $3.89(3.30-7.87)^{* * a}$ \\
\hline & {$[\mathrm{mid}] /[\mathrm{OH}-\mathrm{mid}]$} & 2 & $1.31(1.16-1.77)$ & $3.46(3.10-4.29)^{* * *}$ & $1.73(1.54-2.11) \mathrm{ns}^{\mathrm{a}}$ \\
\hline & {$[\mathrm{mid}] /[\mathrm{OH}-\mathrm{mid}]$} & 4 & $1.51(1.33-1.97)$ & $3.81(3.43-4.58) \mathrm{nt}$ & na \\
\hline & {$[\mathrm{mid}] /[\mathrm{OH}-\mathrm{mid}]$} & 6 & $1.64(1.35-2.31)^{\mathrm{c}}$ & $4.50(3.94-5.60) \mathrm{nt}^{\mathrm{a}}$ & na \\
\hline & {$[\mathrm{mid}] /[\mathrm{OH}-\mathrm{mid}]$} & 8 & na & na & na \\
\hline \multirow[t]{5}{*}{$3 \mathrm{~A} 4$ degluc } & AUCmid/AUCOH-mid tot & & $0.16(0.14-0.21)$ & $0.61(0.54-0.79)^{* * *}$ & $0.04(0.03-0.05)^{* * * a}$ \\
\hline & {$[\mathrm{mid}] /[\mathrm{OH}-\mathrm{mid}] \mathrm{tot}$} & 2 & $0.17(0.15-0.25)$ & $0.64(0.56-0.85)^{* * *}$ & $0.06(0.06-0.08)^{* * * a}$ \\
\hline & {$[\mathrm{mid}] /[\mathrm{OH}-\mathrm{mid}] \mathrm{tot}$} & 4 & $0.20(0.18-0.27)$ & $0.67(0.59-0.86)^{* * *}$ & $0.14(0.06-0.23) \mathrm{ns}^{\mathrm{h}}$ \\
\hline & {$[\mathrm{mid}] /[\mathrm{OH}-\mathrm{mid}] \mathrm{tot}$} & 6 & $0.18(0.15-0.26)$ & $0.65(0.59-0.78) \mathrm{nt}^{\mathrm{a}}$ & na \\
\hline & {$[\mathrm{mid}] /[\mathrm{OH}$-mid]tot } & 8 & na & na & na \\
\hline
\end{tabular}

Data are presented as geometric mean ratios with $90 \%$ confidence intervals

$A U C$ area under plasma concentration-time curve from time 0 to last measurable concentration, $A U C_{24}$ area under the plasma concentration-time curve from time 0 to $24 \mathrm{~h}$, caf caffeine, E3174 losartan carboxylic acid, efa efavirenz, los losartan, met metoprolol, mid midazolam, na not available, $n s$ not significant, $n t$ not tested, $O H$-efa 8-hydroxyefavirenz, $O H$-mid $1^{\prime}$-hydroxymidazolam, $O H$-mid tot total $1^{\prime}$-hydroxymidazolam concentration after deglucuronidation, $\mathrm{OH}$-met $\alpha$-hydroxymetoprolol, $\mathrm{OH}$-ome 5-hydroxyomeprazole, par paraxanthine

$* p<0.025 ; * * p<0.01 ; * * * p<0.001$ (Wilcoxon signed rank test)

$N=16$ unless indicated otherwise: ${ }^{\mathrm{a}} n=15 ;{ }^{\mathrm{b}} n=14 ;{ }^{\mathrm{c}} n=13 ;{ }^{\mathrm{d}} n=10 ;{ }^{\mathrm{e}} n=8 ;{ }^{\mathrm{f}} n=7 ;{ }^{\mathrm{g}} n=6 ;{ }^{\mathrm{h}} n=3$

the highest baseline metabolic ratio was $\mathrm{CYP} 2 \mathrm{~B} 6 * 6$ heterozygous, there was no statistically significant difference between metabolic ratios of wild-type and *6 heterozygous subjects (Fig. 2b).

For CYP2C9, no subject was homozygous for a reduced function allele (*2 or *3). One subject was heterozygous for
CYP2C9*2 and five subjects were heterozygous for CYP2C9*3. Subjects heterozygous for the *3 haplotype had higher metabolic ratios at $6 \mathrm{~h}$, both at baseline (0.13-0.53), and after inhibition (1.20-1.86), and in both conditions they were higher than the metabolic ratios of the other subjects (baseline 0.04-0.11, inhibition 0.20-0.53, Fig. 2c). 
Table 2 Spearman rank correlations between AUC ratios and metabolic ratios at different time points after cocktail administration without and with inhibitors or inducer

\begin{tabular}{|c|c|c|c|c|c|c|c|c|c|c|}
\hline \multirow[t]{2}{*}{ CYP } & \multirow[t]{2}{*}{ Metric } & \multirow[t]{2}{*}{ Time $(\mathrm{h})$} & \multicolumn{2}{|c|}{ All conditions } & \multicolumn{2}{|c|}{ Cocktail alone } & \multicolumn{2}{|c|}{ Cocktail + inhibitors } & \multicolumn{2}{|c|}{ Cocktail + inducer } \\
\hline & & & $\rho_{\mathrm{s}}$ & $p$ value & $\rho_{\mathrm{s}}$ & $p$ value & $\rho_{\mathrm{s}}$ & $p$ value & $\rho_{\mathrm{s}}$ & $p$ value \\
\hline \multirow[t]{4}{*}{$1 \mathrm{~A} 2$} & {$[\mathrm{par}] /[\mathrm{caf}]$} & 2 & 0.917 & $<0.001$ & 0.797 & $<0.001$ & 0.721 & 0.002 & 0.900 & $<0.001^{\mathrm{a}}$ \\
\hline & [par]/[caf] & 4 & 0.935 & $<0.001$ & 0.956 & $<0.001$ & 0.932 & $<0.001$ & 0.804 & $<0.001^{\mathrm{a}}$ \\
\hline & {$[\mathrm{par}] /[\mathrm{caf}]$} & 6 & 0.918 & $<0.001$ & 0.906 & $<0.001$ & 0.893 & $<0.001^{\mathrm{a}}$ & 0.743 & $0.002^{\mathrm{a}}$ \\
\hline & {$[\mathrm{par}] /[\mathrm{caf}]$} & 8 & 0.923 & $<0.001$ & 0.838 & $<0.001$ & 0.853 & $<0.001$ & 0.750 & $0.002^{\mathrm{a}}$ \\
\hline \multirow[t]{4}{*}{$2 \mathrm{~B} 6$} & [efa]/[OH-efa] & 2 & 0.911 & $<0.001$ & 0.559 & 0.024 & 0.719 & $0.004^{\mathrm{b}}$ & 0.636 & $0.011^{\mathrm{a}}$ \\
\hline & {$[\mathrm{efa}] /[\mathrm{OH}-\mathrm{efa}]$} & 4 & 0.942 & $<0.001$ & 0.876 & $<0.001$ & 0.762 & 0.001 & 0.518 & $0.048^{\mathrm{a}}$ \\
\hline & [efa]/[OH-efa] & 6 & 0.975 & $<0.001$ & 0.850 & $<0.001$ & 0.771 & $0.001^{\mathrm{a}}$ & 0.871 & $<0.001^{a}$ \\
\hline & [efa]/[OH-efa] & 8 & 0.965 & $<0.001$ & 0.812 & $<0.001$ & 0.788 & $<0.001$ & 0.729 & $0.002^{\mathrm{a}}$ \\
\hline \multirow[t]{4}{*}{$2 \mathrm{C9}$} & {$[\operatorname{los}] /[\mathrm{E} 3174]$} & 2 & 0.683 & $<0.001$ & 0.706 & 0.002 & 0.429 & $0.004^{\mathrm{f}}$ & 0.380 & $\mathrm{~ns}^{\mathrm{b}}$ \\
\hline & {$[\operatorname{los}] /[\mathrm{E} 3174]$} & 4 & 0.840 & $<0.001$ & 0.762 & 0.001 & 0.529 & 0.035 & 0.589 & $0.021^{\mathrm{a}}$ \\
\hline & {$[\operatorname{los}] /[\mathbf{E 3 1 7 4}]$} & 6 & 0.871 & $<0.001$ & 0.812 & $<0.001$ & 0.754 & $0.001^{\mathrm{a}}$ & 0.602 & $0.017^{\mathrm{a}}$ \\
\hline & {$[\operatorname{los}] /[\mathrm{E} 3174]$} & 8 & 0.848 & $<0.001$ & 0.674 & 0.004 & 0.679 & 0.004 & 0.613 & $0.020^{\mathrm{b}}$ \\
\hline \multirow[t]{4}{*}{$2 \mathrm{C19}$} & [ome]/[OH-ome] & 2 & 0.954 & $<0.001$ & 0.853 & $<0.001$ & 0.156 & ns & 0.936 & $<0.001^{\mathrm{a}}$ \\
\hline & [ome]/[OH-ome] & 4 & 0.959 & $<0.001$ & 0.942 & $<0.001$ & 0.774 & $<0.001$ & 0.546 & $<0.001^{\mathrm{a}}$ \\
\hline & [ome]/[OH-ome] & 6 & & & 0.925 & $<0.001^{\mathrm{b}}$ & 0.886 & $<0.001^{\mathrm{a}}$ & na & \\
\hline & [ome]/[OH-ome] & 8 & & & na & & na & & na & \\
\hline \multirow[t]{4}{*}{$2 \mathrm{D6}$} & {$[$ met $] /[\mathrm{OH}-\mathrm{met}]$} & 2 & & & 0.048 & $\mathrm{~ns}^{\mathrm{e}}$ & na & & 0.564 & $\mathrm{~ns}^{\mathrm{d}}$ \\
\hline & {$[$ met $] /[\mathrm{OH}-\mathrm{met}]$} & 4 & 0.873 & $<0.001$ & 0.815 & $<0.001$ & 0.657 & $\mathrm{~ns}^{\mathrm{g}}$ & 0.736 & $0.003^{\mathrm{b}}$ \\
\hline & [met]/[OH-met] & 6 & 0.879 & $<0.001$ & 0.682 & $0.005^{\mathrm{a}}$ & 0.829 & $0.042^{\mathrm{g}}$ & 0.820 & $<0.001^{b}$ \\
\hline & {$[$ met $] /[\mathrm{OH}-\mathrm{met}]$} & 8 & 0.866 & $<0.001$ & 0.650 & 0.006 & 1.000 & $<0.001^{\mathrm{g}}$ & 0.864 & $<0.001^{\mathrm{b}}$ \\
\hline \multirow[t]{4}{*}{ 3A4 } & {$[\mathrm{mid}] /[\mathrm{OH}-\mathrm{mid}]$} & 2 & & & 0.962 & $<0.001$ & 0.965 & $<0.001$ & 0.743 & $0.002^{\mathrm{a}}$ \\
\hline & {$[\mathrm{mid}] /[\mathrm{OH}-\mathrm{mid}]$} & 4 & & & 0.697 & 0.003 & 0.909 & $<0.001$ & na & \\
\hline & {$[\mathrm{mid}] /[\mathrm{OH}-\mathrm{mid}]$} & 6 & & & 0.687 & $0.01^{\mathrm{c}}$ & 0.796 & $<0.001^{\mathrm{a}}$ & na & \\
\hline & {$[\mathrm{mid}] /[\mathrm{OH}-\mathrm{mid}]$} & 8 & & & na & & na & & na & \\
\hline \multirow[t]{4}{*}{ 3A4 degluc } & {$[$ mid] $] /[$ OH-mid] tot } & 2 & 0.968 & $<0.001$ & 0.921 & $<0.001$ & 0.791 & $<0.001$ & 0.664 & $0.007^{\mathrm{a}}$ \\
\hline & {$[\mathrm{mid}] /[\mathrm{OH}-\mathrm{mid}] \mathrm{tot}$} & 4 & 0.969 & $<0.001$ & 0.900 & $<0.001$ & 0.885 & $<0.001$ & 0.500 & $0.667^{\mathrm{h}}$ \\
\hline & {$[\mathrm{mid}] /[\mathrm{OH}-\mathrm{mid}]$ tot } & 6 & & & 0.894 & $<0.001$ & 0.811 & $<0.001^{\mathrm{a}}$ & na & \\
\hline & {$[\mathrm{mid}] /[\mathrm{OH}-\mathrm{mid}]$ tot } & 8 & & & na & & na & & na & \\
\hline
\end{tabular}

Time points with the highest overall correlation between AUC ratios and metabolic ratios are highlighted in bold

$A U C$ area under plasma concentration-time curve from time 0 to last measurable concentration, caf caffeine, E3174 losartan carboxylic acid, efa efavirenz, los losartan, met metoprolol, mid midazolam, na not available, $n s$ not significant, $O H$-efa 8-hydroxyefavirenz, $O H$-mid 1'-hydroxymidazolam, $\mathrm{OH}$-mid tot total 1'-hydroxymidazolam concentration after deglucuronidation, $\mathrm{OH}$-met $\alpha$-hydroxymetoprolol, $\mathrm{OH}$-ome 5-hydroxyomeprazole, par paraxanthine, $\rho_{s}$ Spearman's correlation coefficient

$N=16$ unless indicated otherwise: ${ }^{\mathrm{a}} n=15 ;{ }^{\mathrm{b}} n=14 ;{ }^{\mathrm{c}} n=13 ;{ }^{\mathrm{d}} n=10 ;{ }^{\mathrm{e}} n=8 ;{ }^{\mathrm{f}} n=7 ;{ }^{\mathrm{g}} n=6 ;{ }^{\mathrm{h}} n=3$

CYP2C19 genotyping identified one poor metabolizer $(\mathrm{CYP} 2 \mathrm{C} 19 * 2 / * 2)$ and four intermediate metabolizers $(\mathrm{CYP} 2 \mathrm{C} 19 * 1 / * 2)$. At baseline, the subject with the poor metabolizer genotype had the highest 4-h metabolic ratio (17.6, Fig. 2d), whereas the metabolic ratios of the IMs (0.5-1.7) did not differ from the metabolic ratios of the EMs $(0.2-2.0)$ with two wild-type alleles $(* 1 / * 1)$.

Four subjects were homozygous and 10 subjects heterozygous for CYP1A2*1F. There was no correlation between genotype and metabolic ratios after induction with rifampicin (data not shown).

\section{Discussion}

In this study, we characterized our previously described phenotyping cocktail in subjects with inhibited or induced CYP activity. Independent of CYP activity, the cocktail was well tolerated and no clinically or statistically significant effects on cardiovascular function or vigilance of the subjects were observed.

Pretreatment with the three CYP inhibitors ciprofloxacin, fluconazole, and paroxetine had a clearly detectable effect on metabolic ratios for all six CYP isoforms and 

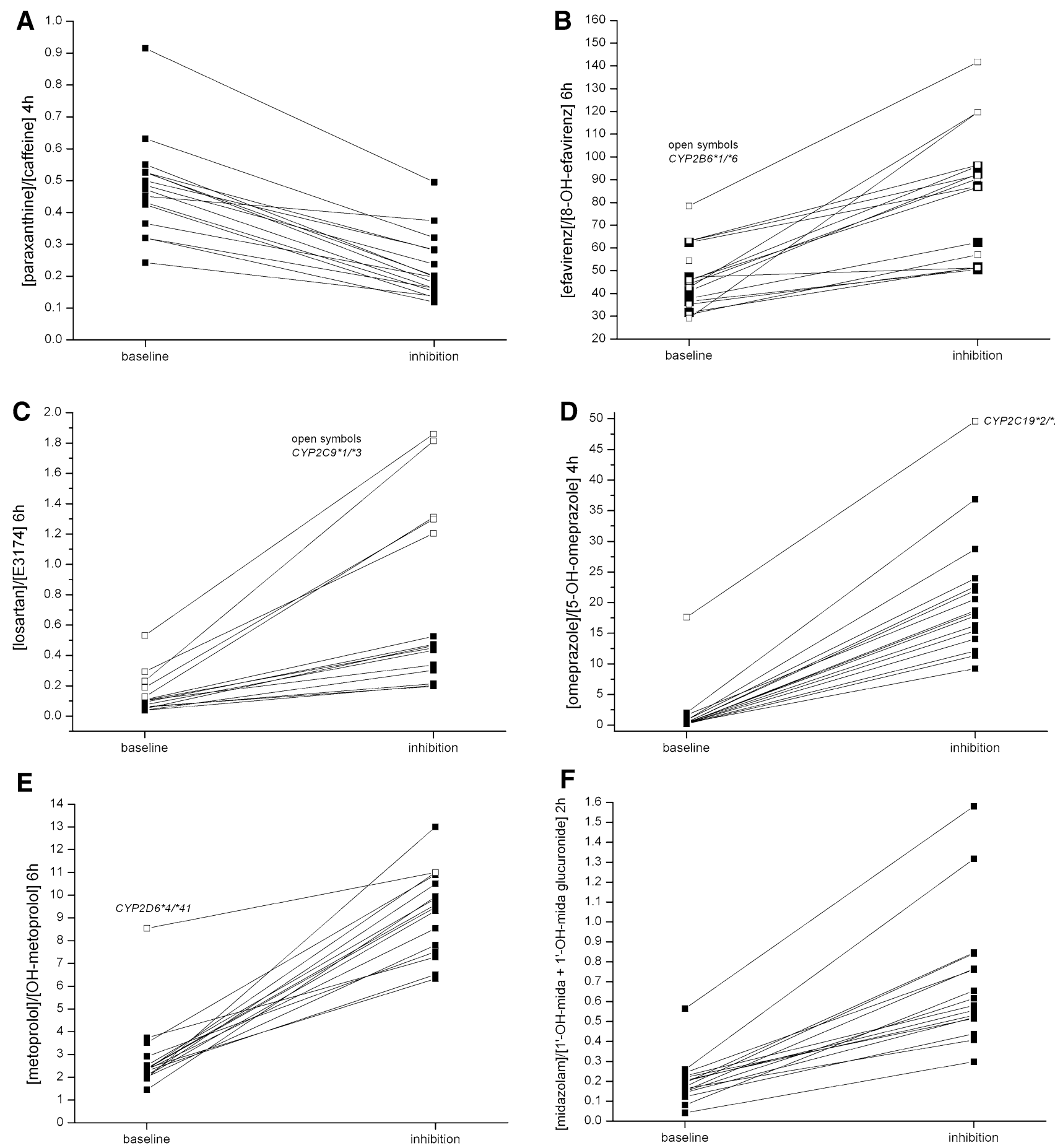

Fig. 2 Intra-individual change of metabolic ratios of the Basel cocktail probe drugs after pretreatment with the CYP inhibitors ciprofloxacin, paroxetine, and fluconazole compared with baseline. CYP inhibition changed the metabolic ratio in every single subject for CYP1A2 (a), CYP2B6 (b), CYP2C9 (c), CYP2C19 (d), CYP2D6 (e), and CYP3A4 (f). Metabolic ratios of subjects with altered function

caused an intra-individual change of the metabolic ratio in almost every study subject.

In contrast to inhibition, CYP induction could not be detected for all the rifampicin-inducible CYP isoforms. For

alleles are shown using open symbols. The CYP2C19 poor metabolizer $(C Y P 2 C 19 * 2 / * 2)$, the CYP2D6 intermediate metabolizer $(C Y P 2 D 6 * 4 / 41)$ and subjects heterozygous for the $C Y P 2 C 9 * 3$ allele had higher metabolic ratios at baseline compared with extensive metabolizer subjects. CYP cytochrome P450 enzyme

CYP2C9, the losartan:E3174 ratio in plasma did not reflect induction. This is in contrast to the situation in urine where pretreatment with rifampicin causes a significant decrease in the losartan:E3174 ratio [3]. The reason for the different 

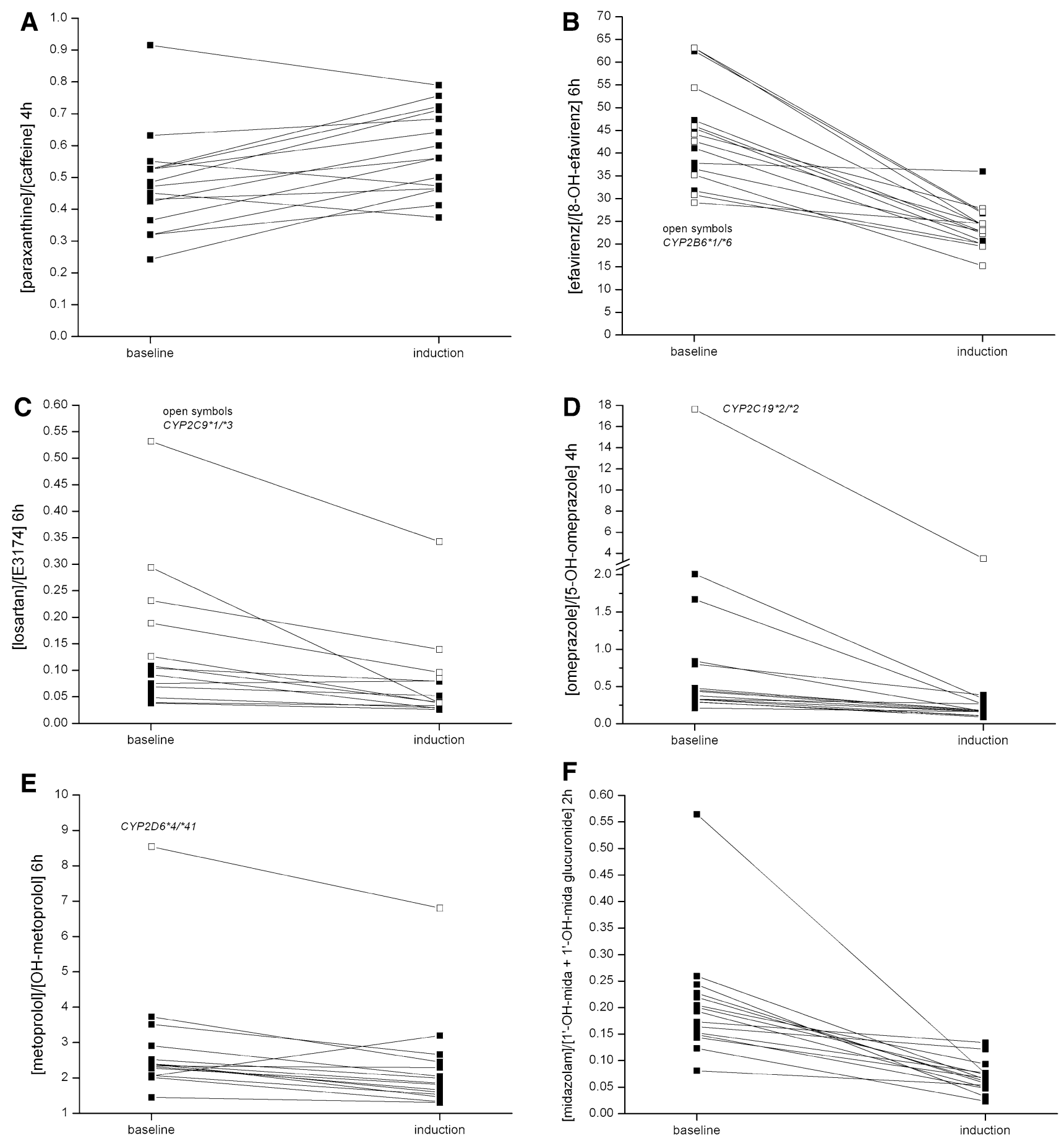

Fig. 3 Intra-individual change of metabolic ratios of the Basel cocktail probe drugs after pretreatment with the CYP inducer rifampicin compared with baseline. A consistent increase of metabolic ratios was observed for CYP1A2 (except for three subjects) (a), and a consistent decrease for CYP2B6 (except for one subject)

behavior of the losartan:E3174 ratio in plasma is that rifampicin not only decreased concentrations of losartan, but also of the E3174 metabolite, most likely by enhancing its further elimination from plasma [21]. The exact

(b), CYP2C19 (d), and CYP3A4 after deglucuronidation (f). The metabolic ratios of CYP2C9 (c), and CYP2D6 (e) only showed small and inconsistent changes after induction. Metabolic ratios of subjects with altered function alleles are shown using open symbols. CYP cytochrome P450 enzyme

pathway of E3174 elimination has not been described. Further metabolism by UDP glucuronosyl transferases has been suggested [21]; however, we did not find an increase in E3174 concentrations after deglucuronidation. Another 
mechanism could be increased elimination by induced efflux pumps. While losartan is transported by p-glycoprotein (pgp), E3174 is not a pgp substrate. There is evidence, however, that other efflux transporters than pgp could be involved in the elimination of E3174 [22]. This is supported by the observation that rifampicin pretreatment increases concentrations of E3174 in urine [3, 4] (and thereby decreases the losartan:E3174 ratio). As there is no easy procedure such as deglucuronidation to assess the total amount of E3174 produced by induced CYP2C9, the losartan:E3174 ratio cannot be recommended for CYP2C9 phenotyping in plasma. Several alternative probe drugs, such as flurbiprofen, warfarin, or phenytoin can be used for CYP2C9 phenotyping. While warfarin and phenytoin have a narrow therapeutic range and require collection of multiple samples [19, 20], flurbiprofen allows limited and minimally invasive sampling strategies [5].

For CYP3A4, the simple midazolam:1'-hydroxymidazolam ratio showed no relevant change after induction because $1^{\prime}$-hydroxymidazolam is further metabolized by UDP glucuronosyl transferases and this phase II reaction is also induced by rifampicin. After induction, glucuronidation may even exceed formation of 1'-hydroxymidazolam and lead to similar metabolic ratios as seen after CYP3A4 inhibition [4]. Therefore, a metabolic ratio that includes both products of the two sequential and inducible reactions will more adequately reflect induction [18]. Because direct quantification of 1'-hydroxymidazolam-glucuronide is difficult, an indirect method was used and samples were pretreated with $\beta$-glucuronidase to allow quantification of the total amount of $1^{\prime}$-hydroxymidazolam produced by the induced CYP3A4. Metabolic ratios using total 1'-hydroxymidazolam concentrations then adequately reflected induced CYP3A4 activity in every study subject. For CYP2B6, however, the efavirenz:8-hydroxyefavirenz ratio without deglucuronidation was sufficient to detect induction.

Induction of CYP1A2 is mediated by the aryl hydrocarbon receptor, which is activated by polycyclic aromatic hydrocarbons and, in contrast to other CYP isoforms, rifampicin only has a weak inducing effect on CYP1A2 [21, 22]. Correspondingly, we only found a small increase in metabolic ratios, which is in line with previous observations $[3,5]$.

CYP2D6 is considered to be a non-inducible CYP isoform and no effects on CYP2D6 phenotyping metrics have been found or reported in previous induction studies $[4,5]$. In our study, we found a small decrease of the metoprolol: $\alpha$-hydroxymetoprolol AUC ratio and an inconsistent decrease of metabolic ratios at different time points. Although $\alpha$-hydroxylation of metoprolol is mainly mediated by CYP2D6, formation of $\alpha$-hydroxymetoprolol by an induced CYP isoform other than CYP2D6 could explain this finding. However, as phenotyping of CYP2D6 is focused on inhibition, which is reliably detected with metoprolol, this does not limit the usefulness of this probe drug for CYP2D6 phenotyping.

Metabolic ratios of the two subjects that were either homozygous for loss-of-function alleles (CYP2C19*2/*2) or had a combination of a reduced function and a loss-offunction allele $($ CYP2D6*4/41) were distinctly higher than metabolic ratios of the other subjects. For the CYP2D6 IM subject, the metabolic ratio was almost the same as the one we found in another subject with the identical genotype in our first cocktail study [7], and it can be expected that subjects with very low metabolic activity of these CYP isoforms can easily be identified with this cocktail. Interestingly, we also found higher losartan:E3174 ratios in plasma for CYP2C $9 * 1 / * 3$ subjects compared with wild-type subjects. This is in line with previous observations where losartan:E3174 ratios in urine also were higher in subjects with the same genotype [23, 24]. However, as already observed in our first study [7], metabolic ratios of subjects only heterozygous for a reduced function allele may also considerably overlap with metabolic ratios of wild-type subjects.

In a recently published cocktail, the same probe drugs for CYP1A2, CYP2C19, and CYP3A4 were used as in our cocktail, while bupropion, flurbiprofen, and dextromethorphan were chosen for CYP2B6, CYP2C9, and CYP2D6, respectively [5]. For bupropion, the absence of an inhibitory effect on CYP2D6 is suggested based on in vitro data and theoretical considerations. For efavirenz, the only other sensitive CYP2B6 substrate listed by the US Food and Drug Administration and the European Medicines Agency besides bupropion, we provided in vivo data demonstrating the lack of interaction with the other components of our cocktail [7]. A common disadvantage for both CYP2B6 probe drugs is their long half-life of 50-70 h, which prolongs wash-out periods when they are used as probe drugs in clinical studies with crossover designs.

Our study has several limitations. While characterization of phenotyping metrics for inhibited and induced CYP activity is an important validation criterion, further validation work is needed. Our study population consisted of young healthy male subjects. While this selection reduces confounding factors, it limits generalizability of the study results to other populations, e.g., female or elderly subjects, which will need to be tested in separate studies. Although the CYP inhibitors and the inducer are recommended compounds for in vivo drug interaction studies, they do not have a strong inhibiting or inducing effect on all the tested CYP isoforms. Therefore, the changes of the phenotyping metric likely will be more pronounced when more potent in vivo inhibitors or inducers can be tested. Another 
limitation is the failure of losartan to reliably indicate CYP2C9 induction, necessitating a modification of the cocktail composition with an alternative probe drug for CYP2C9.

\section{Conclusions}

With this study we provide extensive data for the phenotyping metrics of the Basel cocktail in subjects with inhibited and induced CYP activity. Obtaining such data is an important step in the ongoing validation process of a new phenotyping cocktail. While CYP inhibition could be reliably detected for all CYP isoforms in almost every study subject, detection of CYP induction was more challenging. The losartan:E3174 AUC ratio in plasma did not adequately reflect induced CYP2C9 activity. For CYP3A4, induced CYP activity only was adequately reflected by metabolic ratios using total $1^{\prime}$-hydroxymidazolam concentrations. Although metabolic ratios after CYP inhibition and induction overlap with baseline ratios for many CYP isoforms, intra-individual changes of metabolic ratios are sensitive indicators of altered CYP activity and therefore represent valuable tools for drugdrug interaction studies. The metabolic ratios need to be confirmed for extensive metabolizers, and typical ratios for subjects with genetically altered CYP activity have to be established in separate studies. This will provide important information for future clinical applications of this phenotyping cocktail.

Acknowledgments The authors thank Claudia Blasi and Luisa Baselgia for their assistance with the clinical study and Beatrice Vetter for technical assistance.

Compliance with ethical standards This study was funded by the Division of Clinical Pharmacology and Toxicology, University Hospital Basel.

Christoph Noppen is an employee of Viollier AG, a provider of commercial clinical laboratory services. Adrian Derungs, Massimiliano Donzelli, Benjamin Berger, Stephan Krähenbühl, and Manuel Haschke have no potential conflicts of interest to declare.

The study was approved by the local ethics committee (Ethikkommission Basel) and the national regulatory authorities (Swiss Agency for Therapeutic Products, Swissmedic) and conducted in accordance with the ethical standards of the Declaration of Helsinki. Informed consent was obtained from all individual participants included in the study.

Author contributions Massimiliano Donzelli: designed research, analyzed data, wrote the manuscript.

Adrian Derungs: designed research, performed research, wrote the manuscript.

Benjamin Berger: performed research.

Christoph Noppen: contributed analytical tools, analyzed data.

Stephan Krähenbühl: designed research, analyzed data.

Manuel Haschke: designed research, analyzed data, wrote the manuscript.
Open Access This article is distributed under the terms of the Creative Commons Attribution-NonCommercial 4.0 International License (http://creativecommons.org/licenses/by-nc/4.0/), which permits any noncommercial use, distribution, and reproduction in any medium, provided you give appropriate credit to the original author(s) and the source, provide a link to the Creative Commons license, and indicate if changes were made.

\section{References}

1. Wilkinson GR. Drug metabolism and variability among patients in drug response. N Engl J Med. 2005;352(21):2211-21 (Epub 2005/05/27).

2. Schellens JH, van der Wart JH, Brugman M, Breimer DD. Influence of enzyme induction and inhibition on the oxidation of nifedipine, sparteine, mephenytoin and antipyrine in humans as assessed by a "cocktail" study design. J Pharmacol Exp Ther. 1989;249(2):638-45 (Epub 1989/05/01).

3. Kanebratt KP, Diczfalusy U, Backstrom T, Sparve E, Bredberg E, Bottiger Y, et al. Cytochrome P450 induction by rifampicin in healthy subjects: determination using the Karolinska cocktail and the endogenous CYP3A4 marker 4beta-hydroxycholesterol. Clin Pharmacol Ther. 2008;84(5):589-94 (Epub 2008/07/25).

4. Inui N, Akamatsu T, Uchida S, Tanaka S, Namiki N, Karayama $\mathrm{M}$, et al. Chronological effects of rifampicin discontinuation on cytochrome P450 activity in healthy Japanese volunteers, using the cocktail method. Clin Pharmacol Ther. 2013;94(6):702-8 (Epub 2013/08/27).

5. Bosilkovska M, Samer CF, Deglon J, Rebsamen M, Staub C, Dayer P, et al. Geneva cocktail for cytochrome P450 and P-glycoprotein activity assessment using dried blood spots. Clin Pharmacol Ther 2014;96(3):349-59 (Epub 2014/04/12).

6. Branch RA, Adedoyin A, Frye RF, Wilson JW, Romkes M. In vivo modulation of CYP enzymes by quinidine and rifampin. Clin Pharmacol Ther. 2000;68(4):401-11 (Epub 2000/11/04).

7. Donzelli M, Derungs A, Serratore MG, Noppen C, Nezic L, Krahenbuhl S, et al. The basel cocktail for simultaneous phenotyping of human cytochrome $\mathrm{P} 450$ isoforms in plasma, saliva and dried blood spots. Clin Pharmacokinet. 2014;53(3):271-82 (Epub 2013/11/13).

8. Guidance for industry. Drug interaction studies—study design, data analysis, implications for dosing, and labeling recommendations. Center for Drug Evaluation and Research. Food and Drug Administration, Silver Spring, MD. http://www.fda.gov/ downloads/Drugs/GuidanceComplianceRegulatoryInformation/ Guidances/UCM292362.pdf.

9. Hesse LM, Venkatakrishnan K, Court MH, von Moltke LL, Duan SX, Shader RI, et al. CYP2B6 mediates the in vitro hydroxylation of bupropion: potential drug interactions with other antidepressants. Drug Metab Dispos. 2000;28(10):1176-83 (Epub 2000/09/21).

10. Backman JT, Olkkola KT, Neuvonen PJ. Rifampin drastically reduces plasma concentrations and effects of oral midazolam. Clin Pharmacol Ther. 1996;59(1):7-13 (Epub 1996/01/01).

11. Thorn CF, Aklillu E, Klein TE, Altman RB. PharmGKB summary: very important pharmacogene information for CYP1A2. Pharmacogenet Genomics. 2012;22(1):73-7 (Epub 2011/10/13).

12. Thorn CF, Lamba JK, Lamba V, Klein TE, Altman RB. PharmGKB summary: very important pharmacogene information for CYP2B6. Pharmacogenet Genomics. 2010;20(8):520-3 (Epub 2010/07/22).

13. Van Booven D, Marsh S, McLeod H, Carrillo MW, Sangkuhl K, Klein TE, et al. Cytochrome P450 2C9-CYP2C9. Pharmacogenet Genomics. 2010;20(4):277-81 (Epub 2010/02/13). 
14. Scott SA, Sangkuhl K, Shuldiner AR, Hulot JS, Thorn CF, Altman RB, et al. PharmGKB summary: very important pharmacogene information for cytochrome P450, family 2, subfamily C, polypeptide 19. Pharmacogenet Genomics. 2012;22(2):159-65 (Epub 2011/10/27).

15. Owen RP, Sangkuhl K, Klein TE, Altman RB. Cytochrome P450 2D6. Pharmacogenet Genomics. 2009;19(7):559-62 (Epub 2009/06/11).

16. Cayan F, Ayaz L, Aban M, Dilek S, Gumus LT. Role of CYP2C19 polymorphisms in patients with endometriosis. Gynecol Endocrinol. 2009;25(8):530-5 (Epub 2009/06/06).

17. de Morais SM, Wilkinson GR, Blaisdell J, Nakamura K, Meyer UA, Goldstein JA. The major genetic defect responsible for the polymorphism of $S$-mephenytoin metabolism in humans. J Biol Chem. 1994;269(22):15419-22 (Epub 1994/06/03).

18. Link B, Haschke M, Grignaschi N, Bodmer M, Aschmann YZ, Wenk M, et al. Pharmacokinetics of intravenous and oral midazolam in plasma and saliva in humans: usefulness of saliva as matrix for CYP3A phenotyping. $\mathrm{Br} \mathrm{J}$ Clin Pharmacol. 2008;66(4):473-84 (Epub 2008/06/10).

19. Caraco Y, Muszkat M, Wood AJ. Phenytoin metabolic ratio: a putative marker of CYP2C9 activity in vivo. Pharmacogenetics. 2001;11(7):587-96 (Epub 2001/10/23).
20. Chainuvati S, Nafziger AN, Leeder JS, Gaedigk A, Kearns GL, Sellers E, et al. Combined phenotypic assessment of cytochrome p450 1A2, 2C9, 2C19, 2D6, and 3A, $N$-acetyltransferase-2, and xanthine oxidase activities with the "Cooperstown $5+1$ cocktail”. Clin Pharmacol Ther. 2003;74(5):437-47 (Epub 2003/10/ 31).

21. Maglich JM, Stoltz CM, Goodwin B, Hawkins-Brown D, Moore JT, Kliewer SA. Nuclear pregnane $\mathrm{x}$ receptor and constitutive androstane receptor regulate overlapping but distinct sets of genes involved in xenobiotic detoxification. Mol Pharmacol. 2002;62(3):638-46 (Epub 2002/08/16).

22. Backman JT, Granfors MT, Neuvonen PJ. Rifampicin is only a weak inducer of CYP1A2-mediated presystemic and systemic metabolism: studies with tizanidine and caffeine. Eur J Clin Pharmacol. 2006;62(6):451-61 (Epub 2006/06/08).

23. Yasar U, Dahl ML, Christensen M, Eliasson E. Intra-individual variability in urinary losartan oxidation ratio, an in vivo marker of CYP2C9 activity. Br J Clin Pharmacol. 2002;54(2):183-5 (Epub 2002/09/05).

24. Yasar U, Forslund-Bergengren C, Tybring G, Dorado P, Llerena A, Sjoqvist F, et al. Pharmacokinetics of losartan and its metabolite E-3174 in relation to the CYP2C9 genotype. Clin Pharmacol Ther. 2002;71(1):89-98 (Epub 2002/02/02). 